\title{
Locating the origin of photoelectrons in atomic photoionizaton
}

\author{
I. A. Ivanov* and A. S. Kheifets \\ Research School of Physics and Engineering, The Australian National University, Canberra Australian Capital Territory 0200, Australia
}

(Received 1 September 2011; published 10 February 2012)

\begin{abstract}
We perform a time-delay analysis of the strong field ionization of atomic hydrogen in the tunneling regime. We obtain values for the time delay by solving the time-dependent Schrödinger equation, and use these values as parameters to define the corresponding classical trajectories. We demonstrate that almost all of these trajectories tend to cluster together to a few starting points. The identification of these starting points and their corresponding starting times allows one to answer the question as to when and where the atomic photoionization process actually begins.
\end{abstract}

DOI: 10.1103/PhysRevA.85.021401

PACS number(s): 32.80.Fb, 31.15.ve, 32.30.Rj, 32.70.-n

Presently available experimental techniques, such as attosecond streaking [1,2] and angular attosecond streaking (attoclock) [3,4], make it possible to trace electronic motion in atoms and molecules with a resolution of several attoseconds. This allows one to pose, and experimentally answer, questions which would have seemed rather scholastic merely a decade ago. One such a question, which has recently received considerable attention, is when does atomic photoionization begin? [5]. This question was first raised after the experimental observation of a noticeable time delay between photoelectrons emitted from the $2 s$ and $2 p$ shells in neon [6]. Later, a similar observation of a time delay between the $3 s$ and $3 p$ photoelectrons in argon was reported [7]. The experimental time-delay investigations [6,7] and subsequent theoretical works [8-12] were restricted to the xuv photon energy range and the so-called multiphoton ionization regime. This regime is characterized by values of the Keldysh parameter $\gamma=$ $\omega \sqrt{2 I_{\mathrm{p}}} / F \gg 1$, where $I_{\mathrm{p}}$ is the atomic ionization potential, while $F$ and $\omega$ are the strength and angular frequency of the laser field, respectively. The alternative regime of tunneling ionization in the near-infrared (NIR) photon energy range, with $\gamma<1$, was investigated by Eckle et al. [3,4], who determined experimentally the time needed for an electron to tunnel out of an atom.

In the present Rapid Communication, we also consider the tunneling ionization regime and generalize time-delay theory for this case. We are motivated by the following considerations. The concept of time delay gives us information about a certain combination of initial time and initial coordinate for the trajectory which the center of the ionized wave packet follows [6,8]. If, for simplicity, we assume that the wave-packet trajectory is a straight line, the time delay will be given by the expression $\Delta \simeq t_{0}-r_{0} / v$. In this expression, $v$ is the velocity of the center of the outgoing wave packet, $t_{0}$ is the moment of time when this wave packet is formed, and $r_{0}$ is the coordinate of the event. We recall that, for the tunneling ionization regime, $t_{0}$ is known. As follows from the well-known and extensively tested classical model of tunneling ionization [13], the electron velocity gained in the electromagnetic $(E M)$ field is determined by the value of the vector potential $A\left(t_{0}\right)$ at the moment of ionization. This observation lies at the heart of the attosecond

*igor.ivanov@anu.edu.au streaking technique. We have means, therefore, to determine $t_{0}$ experimentally, and then, knowing the time delay, to unambiguously define $r_{0}$, which is the initial coordinate, or the "birthplace," of the photoelectron at the moment of ionization. Below, we explore this idea more rigorously and present some numerical results. Atomic units are used throughout the Rapid Communication unless otherwise specified.

We consider a photoionization process, driven by a laser pulse, in a hydrogen atom. Below, we will use both classical and quantum descriptions of the electron motion. To simplify our analysis, we consider an experimental geometry in which detectors are placed so that electrons, moving in the forward direction with asymptotic velocity $v$, parallel to the $z$ axis, and impact parameter $R$, are detected at very large times. An asymptotic description of the classical electron trajectories, satisfying the conditions above, can be obtained from Newton's equations of motion in the Coulomb field. Due to the axial symmetry of the problem, we can consider electron motion in a plane, which we choose to be the $(x, z)$ plane. For large time $t \rightarrow \infty$, long after the end of the laser pulse, we can write, for the solution of the classical equations of motion,

$$
\ddot{x}=-\frac{x}{\left(x^{2}+z^{2}\right)^{3 / 2}}, \quad \ddot{z}=-\frac{z}{\left(x^{2}+z^{2}\right)^{3 / 2}},
$$

satisfying the above conditions, the following asymptotic expansions:

$$
x \approx x_{1}, \quad z \approx v t+\frac{\ln t}{v^{2}}+c,
$$

where $\left|x_{1}\right|=R$ and we have omitted terms of order $t^{-1}$. Asymptotic motion is thus determined by the impact parameter $R$ and the constant $c$.

To proceed further, we turn to a quantum-mechanical treatment. We solve the time-dependent Schrödinger equation (TDSE) for a hydrogen atom, driven by a laser pulse with the electric field polarized along the $z$ axis and varying with time for $t \in(-2 T, 2 T)$, and find

$$
F(t)=F_{0} \cos ^{2}\left(\frac{\pi t}{4 T}\right) \cos \omega t .
$$

Here the carrier frequency $\omega=0.0577$ a.u. (corresponding to wavelength $\lambda=790 \mathrm{~nm}$ ), $F_{0}=0.1068$ a.u. (corresponding to a peak intensity of $4 \times 10^{14} \mathrm{~W} / \mathrm{cm}^{2}$ ), and $T=2 \pi / \omega=$ $2.63 \mathrm{fs}$ is the optical cycle period for frequency $\omega$. These values define the Keldysh parameter as $\gamma=0.53$. The field is 

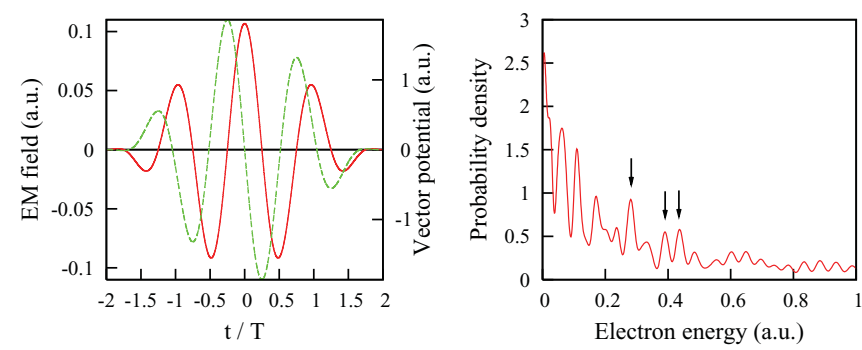

FIG. 1. (Color online) Left: The electric field (red/solid) and vector potential (green/dash) of the laser pulse. Right: The photoelectron energy spectrum. The arrows indicate selected 1 photoelectron energies used in the timing analysis below.

zero outside the interval $(-2 T, 2 T)$, so the total duration of the pulse is $4 T \approx 10.5 \mathrm{fs}$. At the moment of time $t=-2 T$, the hydrogen atom is initially in the ground state. To describe the interaction of the atom and the EM field, we use the velocity gauge $\hat{H}_{\text {int }}(t)=\boldsymbol{A}(t) \cdot \hat{\boldsymbol{p}}$, with $\boldsymbol{A}(t)=-\int_{-2 T}^{t} \boldsymbol{F}(\tau) d \tau$ and the electric field $\boldsymbol{F}(t)$ described above. The time dependence of $F(t)$ and $A(t)$ is shown in the left-hand panel of Fig. 1.

To solve the TDSE, we employ the computational procedure employed in our previous paper [14]. The TDSE is discretized on a spatial grid with a step size of $\delta r=0.05$ a.u. in a box of size $R_{\max }=1500$ a.u. We represent the solution of the TDSE as a partial wave expansion:

$$
\Psi(\boldsymbol{r}, t)=\sum_{l=0}^{l_{\max }} f_{l}(r, t) Y_{l 0}(\theta),
$$

where the summation is restricted to $l_{\max }=20$. This is known [15] to be sufficient to achieve convergence with respect to the number of partial waves for the laser field parameters that we consider. To propagate the wave function (4) on the interval $(-2 T, 2 T)$, we use a matrix iteration method developed in Ref. [16].

We expand the solution of the TDSE for times $t \geqslant 2 T$ on the set of the Coulomb functions (with ingoing boundary conditions)

$$
\Psi(t)=\int a(\boldsymbol{k}) \phi_{\boldsymbol{k}}^{-} e^{-i E_{k} t} d \boldsymbol{k} .
$$

The expansion coefficients $a(\boldsymbol{k})$ determine various ionization probabilities, especially the photoelectron energy spectrum, as shown in the right-hand panel of Fig. 1. More importantly for our purposes, the coefficients $a_{k}$ can be used to study the long-time behavior of the wave packet corresponding to a particular detection geometry. The wave packet describing electrons propagating along the $z$ axis with a particular value of the velocity $v$ and a given value of the impact parameter $R$ can be obtained from the solution $\Psi(t)$ by means of two projection operators, or two "measurements." The first projection operation restricts the range of momentum integration in Eq. (5) to some neighborhood of $\boldsymbol{v}$, where $\boldsymbol{v}$ is directed along the $z$ axis. Another projection restricts the wave function in the perpendicular $(x, y)$ plane to a neighborhood of the point $\rho$ in this plane, such that $\rho=R$. In the case of an ideal (or a von Neumann measurement), this second operation consists of setting the wave function to zero for all $\rho$ outside this neighborhood in the $(x, y)$ plane, while leaving it unchanged for the $\rho$ values inside. For long times, when the "measurements" are performed, we can write such a wave packet as

$$
\tilde{\Psi}(t)=\int_{\Omega} a_{\boldsymbol{k}} \phi_{\boldsymbol{k}}^{-} f(\boldsymbol{\rho}, t) e^{-i E_{k} t} d \boldsymbol{k},
$$

where $\Omega$ is a region in the momentum space containing the point $\boldsymbol{k}=\boldsymbol{v}$, and the function $f(\boldsymbol{\rho}, t)$ may spread in time in the $(x, y)$ plane, but remains peaked at a point in the $(x, y)$ plane corresponding to a given impact parameter. The region $\Omega$ in the momentum space defining integration in Eq. (6) was chosen as follows. We analyze absolute values of the coefficients $a(\boldsymbol{k})$, with $\boldsymbol{k}=\left(0,0, k_{z}\right)$, as a function of $k_{z}$. This function closely follows the pattern shown in the right-hand panel of Fig. 1, which consists of a series of local maxima with energies approximately separated by the carrier frequency $\omega$. We take such a local maximum $k_{z}=v$ as the center of the wave packet, and restrict integration in $k_{z}$ in Eq. (6) to $\left(k_{z} \in \sqrt{v^{2}-\omega}, \sqrt{v^{2}+\omega}\right)$. Thus we obtain wave packets which physically represent electrons contributing to a given peak of the spectrum of above threshold ionization (ATI) and moving in the forward direction.

We are interested in the motion of the center of this wave packet and in matching it to the classical asymptotics in Eq. (2). Similarly to our classical calculation, we can assume that this motion occurs in the $(x, z)$ plane. From Eq. (6), we see that the asymptotic equation for the $x$ coordinate is satisfied automatically. The asymptotic equation for the $z$ coordinate can be obtained by using the saddle-point method with the asymptotic form $\phi_{\boldsymbol{k}}^{-} \propto \exp [i \boldsymbol{k r}+i \gamma(\boldsymbol{r}, \boldsymbol{k})]$, with $\gamma(\boldsymbol{r}, \boldsymbol{k})=$ $k^{-1} \ln (k r+\boldsymbol{k r})$ for the hydrogen scattering states [17,18].

Writing $a_{k}=\left|a_{k}\right| e^{i \delta}$, we obtain the following asymptotic equation for the $z$ coordinate of the center of the wave packet, given by Eq. (6):

$$
z \approx v\left(t-\frac{\partial \delta}{\partial v} \frac{1}{v}\right)+\frac{\ln t}{v^{2}}+\frac{\ln 2 v^{2}-1}{v^{2}} .
$$

The quantity $\Delta=\frac{\partial \delta}{\partial v} \frac{1}{v}$ can be interpreted as the Wigner time delay $[17,18]$. By comparing the classical and quantum asymptotics, we find a relation between the constant $c$ in Eq. (2) and the time delay in Eq. (7).

Knowing the solution of the TDSE, we can determine values of the time delay for our particular geometry. Thus, we can obtain values for the parameter $c$ in the asymptotic classical equation (2) for the $z$ coordinate. This leaves us with one undetermined parameter, $x_{1}$, in the equation for the $x$ coordinate. For different values of the parameter $x_{1}$, we can use asymptotic equations (2) for sufficiently large $t=t_{f}$ (in the calculation we used $t_{f}=5 \times 10^{4}$ a.u.) to compute values of the $x$ and $z$ coordinates at $t=t_{f}$. Together with the known values of the $x$ and $z$ components of the velocity, these values provide us with the initial (or rather final) conditions. By propagating classical equations of motion of an electron in the Coulomb field and the field of the laser pulse defined in Eq. (3) backward in time (we employ the leapfrog method for this purpose), we obtain a one-parameter family (depending on $x_{1}$ as the parameter) of classical electron trajectories. This family has the property that all the trajectories have the 
same high- $t$ asymptotic for the $z$ coordinate, coinciding with the quantum-mechanical asymptotic equation describing the motion of the wave-packet center.

However, not all these trajectories are relevant. From the Keldysh theory of tunneling ionization [19] and its refinements [20-22], we know that the electron velocity at the moment of ionization is small, as high velocities are heavily (exponentially) dampened. The same picture emerges if we use the more physically transparent semiclassical theory of ionization by low-frequency EM radiation developed in Ref. [23], where only the first step of the process-the emergence of the electron into the continuum-is treated quantum mechanically. Describing subsequent evolution as purely classical motion with near zero initial velocity, one obtains photoelectron energy spectra which agree very well with quantum-mechanical results $[13,23]$. The width $\Delta E$ of the kinetic energy distribution for the electrons driven into the continuum by the electromagnetic wave can be estimated using the uncertainty relation as $\Delta E=h / \Delta t$ [23], where $\Delta t=\gamma T, \gamma$ is the Keldysh parameter, and $T$ is the duration of an optical cycle. The parameter $\Delta t$ is the tunneling time introduced in Ref. [19]. It is the time required for an electron to tunnel out from the atom. For the field parameters we use, we obtain $\Delta E \approx 0.1$ a.u. Correspondingly, the velocity distribution of the electrons emerging into the continuum should peak around zero velocity with a characteristic width of $\Delta v \approx 0.4$ a.u. A quantum-mechanical treatment [20] shows that this simple estimate is, in fact, accurate for the width of the distribution of the lateral (i.e., perpendicular to the EM field polarization direction) electron velocities. The distribution of the longitudinal velocities is also peaked at zero velocity, but its width is generally larger [20].

Therefore, we can restrict our one-parameter family of classical trajectories to those trajectories for which the initial value of the lateral velocity is lower than the above estimate. We can also expect that an electron will emerge into the continuum not far from the nucleus and so the absolute value of its initial $z$ coordinate is not too large.

Values of initial velocity and coordinates for a given trajectory can be determined as follows. We trace the velocity change along each trajectory and look for the minima of its absolute value. If, for all the minima on a given trajectory, the lateral velocity exceeds the value $v_{\max }=\Delta v$, we discard that trajectory. We also discard the trajectories for which there are minima with absolute values of the initial lateral velocity below $v_{\max }$, but where the corresponding absolute value of the $z$ coordinate is greater than some $z_{\max }$.

The results presented below were obtained using $z_{\max }=$ $12 \mathrm{au}$. The particular numerical values of the parameters $z_{\max }$ and $\Delta v$ are, in fact, not very important, as their role is merely to define a filtering procedure which discards those classical trajectories which most strongly violate our expectations, based on the theory of tunneling ionization. Similarly, using different filtering criteria to discard irrelevant electron trajectories does not alter the conclusions presented below, as long as the criteria comply with the tunneling ionization theory. We checked this fact, varying parameters $z_{\max }$ and $\Delta v$ for the filtering criteria we described above, and performing the calculations employing different criteria, discarding the classical trajectories for which not lateral but total electron velocity at the moment of ionization event exceeds some critical value.

For each classical trajectory which passes the filtering procedure, we obtain a point $t_{0}$ where the minimum of the absolute value of the velocity was attained, and corresponding values of $x_{0}=x\left(t_{0}\right)$ and $z_{0}=z\left(t_{0}\right)$. It is natural to interpret $t_{0}$ as the moment of time at which ionization occurs, whereas $x_{0}$ and $z_{0}$ define the spatial coordinates of this event.

To illustrate our findings, we select several peak photoelectron energies, viz., $E=0.283,0.391$, and 0.435 a.u.; these are marked by arrows in the right-hand panel of Fig. 1. The time-delay values obtained from the TDSE solution for these energies are $\Delta=535,421$, and 371 as, respectively. The corresponding values of $x\left(t_{0}\right)$ and $z\left(t_{0}\right)$, for those trajectories which have passed the filtering procedure, are plotted in Fig. 2.

Upon inspection of this figure, it becomes immediately obvious that almost all of the trajectories which satisfy the tunneling ionization criteria start at about the same moment of time, viz., $t_{0} \simeq 0.4 T$, with the initial values $z_{0}$ distributed sharply. This indicates that our interpretation of $t_{0}$ and $z_{0}$ as the time and initial $z$ coordinate of the ionization event may be a meaningful one.

The clustering of the classical trajectories can be visualized even better if we plot the density of the trajectories with given $x_{0}, z_{0}$. We define this density as a number of trajectories with
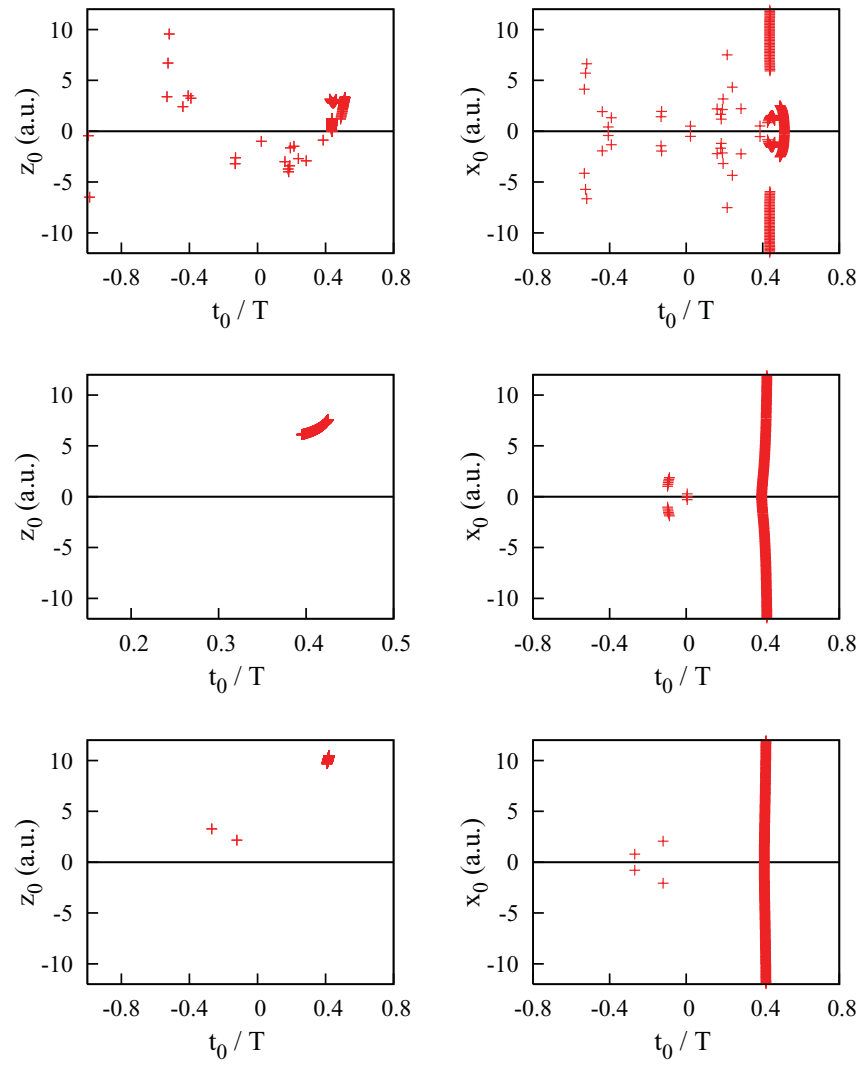

FIG. 2. (Color online) Distributions of initial values $z_{0}=z\left(t_{0}\right)$ (left-hand column) and $x_{0}=x\left(t_{0}\right)$ (right-hand column) for classical trajectories corresponding to photoelectrons moving in a forward direction, with energies $E=0.283$ a.u. (upper row), 0.391 a.u. (middle), and 0.435 a.u. (lower row). 


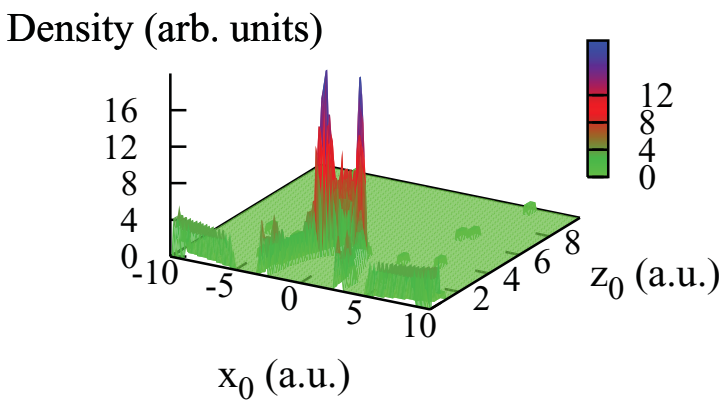

FIG. 3. (Color online) Density of the classical trajectories with initial coordinates $x_{0}, z_{0}$ for the electron energy $E=0.283$ a.u.

given $\left(x_{0}, z_{0}\right)$ per unit area in the $\left(x_{0}, z_{0}\right)$ plane, which have passed the filtering procedure. Thus defined density, shown in the Fig. 3 for the electron energy $E=0.283$ a.u., exhibits a sharp maximum in the $z$ direction.

We note further that the value of $t_{0}$, which we interpret as the moment when photoionization "begins," agrees well with what one would expect on the basis of the classical model [13]. This model predicts that the photoelectron velocity gained in the EM field is determined by the value of the vector potential $A\left(t_{0}\right)$ at the moment of ionization. From Fig. 1, we observe that $A\left(t_{0} \simeq 0.4 T\right) \approx 1$ a.u., which falls into the range of the final velocities that we presently consider (from 0.75 to 0.93 a.u.).

Figure 2 shows that $z_{0}$ grows with electron energy. This growth can be explained in the following way. As a rough approximation, we can picture electron motion in the $z$ direction as a straight line. The value of the $z$ coordinate at the moment of ionization is then $z_{0}=v\left(t_{0}-\Delta\right)$, where $\Delta$ is the time delay which decreases with energy.
If we plug the values of the time delays reported above into this formula, together with $t_{0} \approx 0.4 T$, we obtain $z_{0}=$ $16.5,23.1$, and 26.7 a.u. for the energies of $0.283,0.391$, and 0.435 a.u., respectively. The absolute values of $z_{0}$ derived from the straight-line trajectories are inaccurate because of the crude character of this approximation. However, these values demonstrate the same characteristic increase with energy that is seen in Fig. 2.

Plots for the initial values of the $x$ coordinate in Fig. 2 are less informative. They again tell us that trajectories start at $t_{0} \approx 0.4 T$.

To summarize, we propose a procedure which offers us a conceptual framework allowing us to discuss questions such as when and where does atomic photoionization actually begin? As always, when trying to describe a quantum-mechanical phenomenon using classical language, we have to rely on a classical tool. In this Rapid Communication, such a tool is classical trajectory analysis, using additional information about the time delay provided by the quantum-mechanical TDSE calculation. By selecting trajectories which comply with the classical theory of tunneling ionization, we can unambiguously define the moment of time when ionization occurs and the spatial coordinates of this event. Thus defined, the time of ionization $t_{0}$ agrees well with what one would expect from a purely classical model [13]. The success of the latter model in describing phenomena such as ATI, multiphoton two-electron ejection, or high harmonic generation, tells us that classical concepts, such as the moment of time when an ionization event occurs, may be extremely useful. In the present Rapid Communication, we introduced another such concept-localization of the ionization event in space. We hope this localization may be determined experimentally in future attosecond experiments.
[1] A. Baltuška, T. Udem, M. Uiberacker, M. Hentschel, E. Goulielmakis, C. Gohle, R. Holzwarth, V. S. Yakovlev, A. Scrinzi, T. W. Hänsch, and F. Krausz, Nature (London) 421, 611 (2003).

[2] R. Kienberger, E. Goulielmakis, M. Uiberacker, A. Baltuska, V. Yakovlev, and F. Bammer, Nature (London) 427, 817 (2004).

[3] P. Eckle, M. Smolarski, P. Schlup, J. Biegert, A. Staudte, M. Schoffler, H. G. Muller, R. Dorner, and U. Keller, Nat. Phys. 4, 565 (2008).

[4] P. Eckle, A. N. Pfeiffer, C. Cirelli, A. Staudte, R. Dorner, H. G. Muller, M. Buttiker, and U. Keller, Science 322, 1525 (2008).

[5] H. W. van der Hart, Science 328, 1645 (2010).

[6] M. Schultze, M. Fiess, N. Karpowicz, J. Gagnon, M. Korbman, M. Hofstetter, S. Neppl, A. L. Cavalieri, Y. Komninos, T. Mercouris, C. A. Nicolaides, R. Pazourek, S. Nagele, J. Feist, J. Burgdörfer, A. M. Azzeer, R. Ernstorfer, R. Kienberger, U. Kleineberg, E. Goulielmakis, F. Krausz, and V. S. Yakovlev, Science 328, 1658 (2010).

[7] K. Klünder, J. M. Dahlström, M. Gisselbrecht, T. Fordell, M. Swoboda, D. Guénot, P. Johnsson, J. Caillat, J. Mauritsson, A. Maquet, R. Taïeb, and A. L'Huillier, Phys. Rev. Lett. 106, 143002 (2011).

[8] A. S. Kheifets and I. A. Ivanov, Phys. Rev. Lett. 105, 233002 (2010).
[9] A. S. Kheifets, I. A. Ivanov, and I. Bray, J. Phys. B 44, 101003 (2011).

[10] I. A. Ivanov, Phys. Rev. A 83, 023421 (2011).

[11] S. Nagele, R. Pazourek, J. Feist, K. Doblhoff-Dier, C. Lemell, K. Tôkési, and J. Burgdörfer, J. Phys. B 44, 081001 (2011).

[12] L. R. Moore, M. A. Lysaght, J. S. Parker, H. W. van der Hart, and K. T. Taylor, Phys. Rev. A 84, 061404 (2011).

[13] P. B. Corkum, Phys. Rev. Lett. 71, 1994 (1993).

[14] I. A. Ivanov, Phys. Rev. A 82, 033404 (2010).

[15] A. N. Grum-Grzhimailo, B. Abeln, K. Bartschat, D. Weflen, and T. Urness, Phys. Rev. A 81, 043408 (2010).

[16] M. Nurhuda and F. H. M. Faisal, Phys. Rev. A 60, 3125 (1999).

[17] E. P. Wigner, Phys. Rev. 98, 145 (1955).

[18] C. A. A. de Carvalho and H. M. Nussenzveig, Phys. Rep. 364, 83 (2002).

[19] L. V. Keldysh, Sov. Phys. JETP 20, 1307 (1965).

[20] A. M. Perelomov and V. S. Popov, Sov. Phys. JETP 25, 482 (1967).

[21] F. H. M. Faisal, J. Phys. B 6, L89 (1973).

[22] H. R. Reiss, Phys. Rev. A 22, 1786 (1980).

[23] P. B. Corkum, N. H. Burnett, and F. Brunel, Phys. Rev. Lett. 62, 1259 (1989). 